\title{
Probing for local activity-related modulation of the infrared backscattering of the brain cortex.
}

${ }^{1}$ Gabriele E.M. Biella, ${ }^{2,3}$ Stefano Trevisan, and ${ }^{3, *}$ Mario E. Giardini

\author{
${ }^{1}$ Institute of Molecular Bioimaging and Physiology - IBFM \\ National Research Council - CNR - Italy \\ ${ }^{2}$ Dipartimento di Elettronica, Università di Pavia, Italy \\ ${ }^{3}$ National Institute for Physics of Matter - INFM, Italy
}

* to whom all correspondence should be addressed.

This is the peer reviewed version of the following article: Probing for local activityrelated modulation of the infrared backscattering of the brain cortex, Biella, G. E. M., Trevisan, S. \& Giardini, M. E.,J. Biophot. 2 (2009) 588-595, which has been published in final form at http://onlinelibrary.wiley.com/doi/10.1002/jbio.200810067/abstract . This article may be used for non-commercial purposes in accordance with Wiley Terms and Conditions for Self-Archiving 


\begin{abstract}
The possibility to measure the metabolic activity of the brain cortex, with submillimetre spatial and subsecond temporal resolution, would open enticing scenarios in addressing basic issues on the relation between different structural components of brain signal processing, and in providing an operational pathway to interaction with (dis)functional signal patterns. In the present article, we report the description of a simple system that allows the detection of the minute changes that occur in the optical backscattering of the cortex as a metabolic response to external stimuli. The simplicity of the system is compatible with scalability to an implantable probe. We validate the system on an animal model, and we propose an algorithm to extract meaningful data from the measured signal. We thus show the detection of individual haemodynamic cortical responses to individual stimulation events, and we provide operational considerations on the signal structure.
\end{abstract}




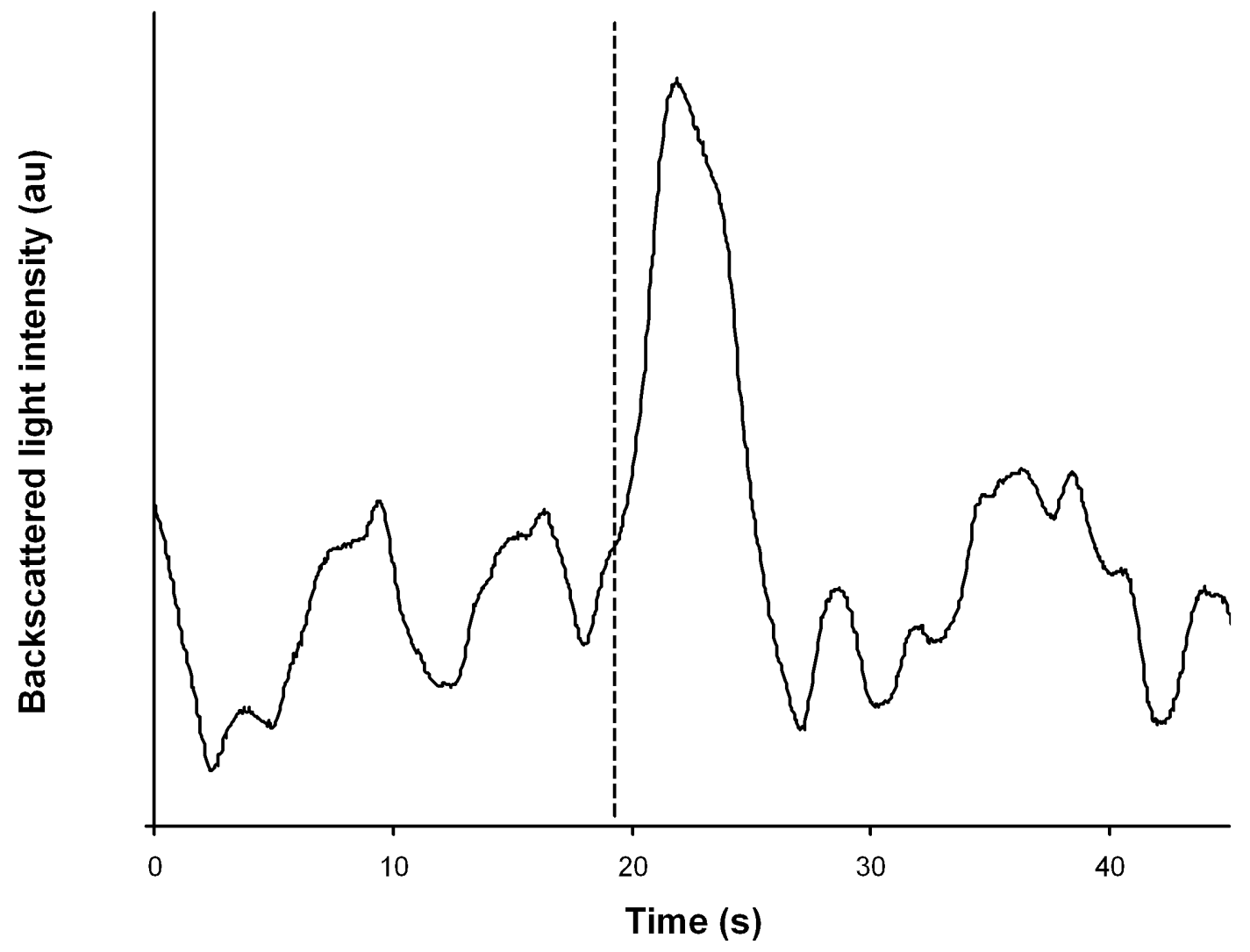

\section{ABSTRACT FIGURE LEGEND}

Change in the optical backscattering of the brain cortex area associated with the hind paw of a rat, as a response of a single stimulus (electrical pulse, dotted line) applied to the paw. 
SHORT TITLE:

G.E.M. Biella, S. Trevisan and M.E. Giardini: Activity and infrared backscattering of the brain cortex.

\section{KEYWORDS}

infrared backscattering, detection of neuronal activity, optical detection, biomedical transducer, rat

\section{PACS}

42.81.Pa, 07.57.Ty, 87.19.1m, 87.64.Cc, 87.85.Ox 


\section{INTRODUCTION}

From an optical point of view, most biological tissues can be considered as absorbing matrices, where a number of inhomogeneities, acting as light scatterers, are present [1]. When collimated near infrared (NIR) light is injected into the tissue and the collimated transmission is observed, light losses are mainly due to scattering rather than to absorption [2]. In the biomedical field, the spectral analysis of the backscattered light is usually referred to as NIRS (Near Infra-Red Spectroscopy) [3].

In the NIR spectral region, the tissue optical absorption is dominated by blood red cell haemoglobin, that carries oxygen by fixing it in the oxygenated form (oxyhaemoglobin) in the lungs and releasing it into the tissues, thus reverting to the deoxygenated form (deoxyhaemoglobin). As the two forms present significantly different NIR absorption spectra, the overall optical properties of the tissues depend therefore on the available oxygen [4].

Biological tissues regulate oxygen consumption by fine adjustments of local mechanical and chemical parameters (e.g. perivascular muscle contractility acting on precapillary arteriole diametre, or oxygen extraction rate). The brain shows highest oxygen consumption rates and metabolic levels than of all biological tissues, closely related to both neural and glial activities [5] in either spontaneous ongoing or evoked regimes. The energy required is primarily conveyed by oxygen and glucose, which act as limiting factors on brain performance: the brain's information-processing capacity is limited by the amount of energy available. [6].

This fact commits with the original hypothesis of the close direct relation between oxygen consumption and brain activity and provides a rationale to functional brain imaging techniques (such as functional Magnetic Resonance - fMRI) in experimental and 
clinical studies [7]. As a rule, higher performance mutually relates to higher blood flow, consequent to higher metabolic requests. A further proof, in negative, is that high rate of oxygen consumption even in resting conditions accounts for the severe nervous damages in anoxia or low oxygenation levels, yet with few exceptions [8].

Brain tissue NIRS would thus be a fast and reliable technique, capable of detecting local oxygen supply fluctuations due to specific activation states, either spontaneous [9] or not $[10,11]$.

Beyond NIRS, a number of other spatially resolved techniques [12, 13] allow for living brain functional mapping. They range from multispectral optical imaging, to voltage sensitive dye imaging, to in-vivo two-photon or multi-photon microscopy. In such scenario, NIRS offers the potential to be performed either noninvasively or through a minimally invasive approach, thus constituting both a crucial single-point and a topo/tomographic complement or, potentially, a tool per se, to diagnose the biochemicalmetabolic substrate of the neural activity.

However, NIRS is burdened with a number of drawbacks, not least the poor spatial localisation, whether intended as spatial resolution in diffuse optical tomography (DOT) or single-point field-of-view in spot measurements. Moreover, as detailed further on, NIRS is imposing increasing demands on specific oxygenation modelling, requiring a reconsideration of brain oxygenation dynamics with respect to current knowledge.

Still, commercial noninvasive (transcranial) dedicated NIRS instruments [14, 15] display performances on optical fluctuations related to brain oxygen consumption activity on the hundred milliseconds time scale and with a few centimetre lateral localisation. However, brain electrical activity may lie on much shorter time scales (in the few milliseconds range) and, most of all, on variable extensions of the involved neural networks (from tens of microns to centimetres). Thus, the question on whether the 
underlying metabolic activity and subsequent optical modulation may yield significant functional information, if considered on such time-space scales, is yet to be approached, and significant work is being done in the literature in this direction $[16,17]$.

This scenario suggests that spatially and temporally local activity-related optical data measured directly on the brain cortex itself would deliver more correct parameters, at least for the definition of a reference framework. Furthermore, if accessible using implantable probes, such data could potentially be used for real-time functional diagnostics and detection.

Experimental optical measurements by direct vascular imaging on the brain cortex $[18,19]$ are already performed during surgery. As for NIRS-based intrasurgical measurements, that can access the size and time scales required and that, unlike imaging, offer potential for long-term implantable devices, the current state of the art relies on a time average of a high number of individual signals [19], thus losing any information that may pertain to a punctual analysis of the actual temporal behaviour.

In this paper, we present a NIRS measurement setup that allows for direct and local activity-related modulation of the brain cortex infrared backscattering, aiming for a singlepoint (non-tomographic) measurement, scalable to implantable applications. In particular, we target the system towards a mesoscopic-scale measurement of the timing of singleevent metabolic activity with relation to neural activation, as this is expected to carry major information on data processing within the brain [20]. We show that a simple system, compatible with integration processes required for implant development, can deliver signals that do not require averaging for significant data to be obtained. We report measurements in a model animal system, and we perform an analysis of such measurements for consistent data extraction. 


\section{EXPERIMENTAL}

\section{1 - The NIRS system}

The system was built around the specific requirement of evaluating the feasibility of local and fast intrasurgical stimulus-related NIRS response measurements, both for reference data production and, most importantly, for performance evaluation of potentially implantable micro-engineered NIRS probes, for the prospective detection of the metabolic activity related to neural data processing.

It consisted of an emitter and a detection probe (Figure 1.a,b), both positioned on the cerebral cortex. Micro-engineered optical probe heads have been functionally simulated by using fibre optical coupling towards the brain cortex tissue. Moreover, as to allow potential miniaturisation, the front-end electronics has been simplified to the maximum extent compatible with the intended use. Ancillary acquisition instruments allowed for signal recording.

As emitter, we employed a $950 \mathrm{~nm}$ LED (Siemens LD274) NIR source. Though somewhat close to the first infrared absorption band of water, such wavelength, on which oxy- and deoxyhaemoglobin exhibit a very high differential optical contrast, has the advantage of allowing a very effective and simple shielding towards environment light of a matching detector, using readily available polymeric optical filter materials.

The LED was driven to emit continuous-wave infrared light, using a batterypowered low noise linear regulator, free of power-supply induced light modulation. We pigtailed it by simple proximity, coupling it to a $15 \mathrm{~cm}$ polymeric optical fibre. Namely, we exposed the LED chip by drilling into the epoxy LED chamber, and we glued the fibre into the cavity, interposing a refractive index-matching gel. We encapsulated in 
thermorestringent tubing the LED-to-fibre joint region, for optical shielding and mechanical strengthening. The probe was sufficiently stiff to allow positioning without any further mechanical reinforcement.

As detector, we used a PIN photodiode (Siemens BPW34F). Such detector was shielded from ambient light by a highly efficient epoxy encapsulating bandpass optical filter, with a transmission window centred on $950 \mathrm{~nm}$. Again, a $15 \mathrm{~cm}$ polymeric optical fibre was pigtailed directly on the filter using a suitable optical adhesive and the fibredetector assembly, encapsulated on the photodiode-to-fibre joint, could be positioned without any additional mechanical stiffening.

The photodiode signal was preamplified by an extremely simple preamplifier stage, whose schematic diagram is reported (Fig 1.c). Such stage was configured for a bandpass response, and accessed (without electronic post-equalization) time scales from $100 \mathrm{~ms}$ to 5 s. The preamplifier, battery-powered, was housed in an electrically and magnetically shielded enclosure, connected to the photodiode by a short $(50 \mathrm{~cm})$ shielded cable.

Both the source and the detector fibres were polymeric light-guides with a $1 \mathrm{~mm}$ core diameter. We chose this size as optimally matching the expected activated areas on the cerebral cortex for the validation protocols employed.

As, within the experimental target and applicational outlook, we aimed to metabolic activity ("optical spike") timing, both a single wavelength technique and the system bandwidth have been deemed sufficient in a cost-benefit evaluation. With simple modulation techniques [21], no obstacle can be envisaged in extending the method to multiple wavelengths and, using known noise reduction related to synchronous detection, to faster time response, e.g. to perform fast quantitative haemoglobin analysis, at the cost of an increased electronic complexity and size. 


\subsection{The animal model and the recording apparatus}

Nine rats (Sprague-Dawley, Charles-River, Calco, LC, Italy) weighing 350-400 gm were used. The animals were maintained with regulated $16 \mathrm{hrs}$ light- $8 \mathrm{hrs}$ dark cycles, food and water ad libitum.

All the animals have been treated along the Italian and European Laws on animal treatment in Scientific Research (Italian Bioethical Committee, Law Decree on the Treatment of Animals in Research, 27 Jan 1992, No 116). The National Research Council, where the experiments have been performed, adheres to the International Committee on Laboratory Animal Science (ICLAS) on behalf of United Nations Educational, Scientific and Cultural Organizations (UNESCO), the Council for International Organizations of Medical Sciences (CIOMS) and the International Union of Biological Sciences (IUBS). As such, no protocol-specific approval was required.

In addition, all experiments were carried out in strict observance of the ethical guidelines of the International Association for the Study of Pain [22].

The rats underwent preliminary barbiturate anaesthesia for the surgical experimental preparation (Pentobarbital $2.5 \mathrm{mg} / \mathrm{kg} / \mathrm{i} . \mathrm{p}$.). The jugular vein and the trachea were delicately desheathed and cannulated to gain, respectively, a drug delivery access and the connection to the forced anaesthesia-ventilation device. The rats were then mounted on a stereotaxic frame and the head was firmly fixed to the frame by usual stereotactical constraints (Narishige, Tokyo, Japan). An electronically regulated thermal bed maintained the rat temperature at $37.5^{\circ} \mathrm{C}$. The scalp was delicately removed from the parietal bones and the skull planum was prepared for the placement of both the NIRS probes and the EEG electrodes. The stereotactical measures were chosen along the indications of the PaxinosWatson stereotactic brain atlas [23]. Before the placement of the probes the rats were 
paralyzed by intravenous gallamine thriethiodide $(20 \mathrm{mg} / \mathrm{kg} / \mathrm{h})$ injection and connected to the respiratory device delivering (1stroke/s) an Isoflurane ${ }^{\circledR} 2.5 \% 0.61 / \mathrm{min}$ in Oxygen 0.15 $0.2 \mathrm{l} / \mathrm{min}$ gaseous mixture. Curarisation was maintained stable throughout the whole experiment by gallamine supplementary injections to avoid spurious movement interference.

\subsubsection{Complementary EEG recordings}

We performed EEG recordings to check continuously the anaesthesia levels. The recordings were carried out from monolateral recording (four plus one reference) electrodes in fronto-occipital sequence, to control the anaesthesia depth (Frontal cortex AP [Bregma] +3.0, ML -2.0; Fronto-Parietal Cortex [Somato-sensory primary Cortex] [Bregma] AP -1, ML - 2.5; Mid-Parietal Cortex [Hippocampus] AP [Bregma] -4.3, ML 2.5; Parieto-Occipital [Bregma] AP -10, ML - 2.0). One of the EEG electrodes (the second derivation) was mirror-like and complementary to the contralateral somatosensory primary cortex from where the NIRS cortical recordings were performed.

For signal EEG amplification and data recordings a 32 channel Cheetah Data Acquisition Hardware was used (Neuralynx, MT, USA) at $32 \mathrm{kHz}$ sampling frequency. Electrophysiological EEG data were digitized and recorded with filtering low-high pass bands of $475 \mathrm{~Hz}-1 \mathrm{~Hz}$ respectively. Trace visualization was achieved by multitrace digital oscilloscopes (National Instruments, Milan, Italy). The data stored were analysed off-line both by Matlab ${ }^{\mathrm{TM}}$ and by locally developed software.

\subsubsection{NIRS recording preparation}

For the NIRS recordings, two $3 \mathrm{~mm}^{2}$ windows were drilled on one side of the skull to gain access to the brain structures (cortical surfaces) lying below. The first hole (- 0.1 to - 
$3 \mathrm{~mm}$ AP [Bregma] and 0.1 to $3 \mathrm{~mm} \mathrm{ML)}$ was drilled over the virtual centre of the oblong hind limb cortical projection map (AP [Bregma] $+0.3 \mathrm{~mm}$ to $-2.3 \mathrm{~mm}$; ML $-0,1$ to -3.5 ) of the Somato-Sensory Primary Cortex (SSI). The second window was drilled posteriorly in regions of visual signal processing, well distinct from the somatosensory regions (AP [Bregma] $-6.0 \mathrm{~mm}, \mathrm{ML}-3.8 \mathrm{~mm}$ ). After the removal of the excised bone tiles, the dura mater was delicately cleaned, under surgical binocular microscope supervision. After positioning on the cortical surfaces, the probes were separately advanced in $2 \mu \mathrm{m}$ steps until proximal to the meninges. Each probe was driven by a separate linear stepper device (Transvertex, Stockholm, Sweden and Narishige, Tokyo, Japan). The "somatosensory" recording probe was preliminarily positioned at the cortical dural surface. The other probe was then lowered upon the local surface on the sensory silent region. The source probe was then switched on, and the LED driven as to tune the power on the fibre tip to the dynamic range of the detection system, and namely on the order of $10 \mu \mathrm{W}$ (corresponding to a power density of $13 \mu \mathrm{W} / \mathrm{mm}^{2}$ ). After the probe placements, the EEG electrodes were positioned.

\subsubsection{Stimulation protocol}

We delivered bipolar stimuli $(10 \mathrm{~ms}, 5 \mathrm{~mA})$ with $25 \mathrm{~s}$ interpulse spacing. We connected the stimulating device to an optical isolator unit, to avoid backward current effects, and connected the isolator by an opportunely length wire to the stimulating needlelike electrodes. We then inserted gently the electrode couple into the volar surface of the posterior paw contralateral to the cortical NIRS recording probe. No spurious movements were locally generated during the stimulating periods along the paw but for a gentle finger flexion synchronous to the stimulus delivery. 


\subsection{The measurement protocol}

The whole EEG+NIRS system was located inside a Faraday cage with all batteryoperated equipment (source probe, detector probe and preamplifier), a surgical illuminator and a surgical microscope. All ancillary instruments (digital oscilloscope - Yokogawa DL1400 - for NIRS probe signal acquisition, thermal regulator for the bed, power supply for the illuminator) were located outside the cage.

The NIRS probes were placed, and the signal was kept under observation until a stationary condition was reached and consistently maintained.

Sequences of up to 10 stimuli, have been applied to the paw, and the corresponding NIRS signal picked up by the detector has been recorded with $20 \mathrm{~Hz}$ sampling frequency. After every sequence, sufficient time was allowed for full recovery and stabilization of the stationary conditions for all measured signals, typically requiring 10-20 minutes.

EEG recordings, performed as control for the depth of anaesthesia, fluctuated in the range from 0.5 to $12 \mathrm{~Hz}$, indicating sound anaesthesia levels

\section{RESULTS AND DISCUSSION}

In Figure 2.a the NIRS signal in presence of five stimulus pulses is reported. A strong periodic oscillation is clearly visible (Fig. 2 insert), and can be ascribed to local modulation of the optical backscattering due to the periodic oxygenation and deoxygenation of blood during the ventilation cycle.

In order to separate the respiratory-synchronous from the asynchronous components, the signal has been modellised locally, on a sliding time window with duration of one respiratory cycle, as a sinusoid offset by a linear background, i.e. 


$$
S(t+\tau)=A(t)+B(t) \tau+C(t) \cos (D \tau+E(t))
$$

where $t$ is the time, $S$ is the signal, and $\tau$ varies from 0 to the (known) respiratory cycle duration. Indeed D and E are known from the respiratory frequency and initial phase, while A, B and C may be expected to carry information, and have been extracted by LevenbergMarquardt regression [24] on the given time window for $\tau$. Typical results are reported in Fig. 2.b, 2.c, 2.d. In order to account for random fluctuations of the mechanical ventilator, the phase $\mathrm{E}(\mathrm{t})$ has been fitted alongside $\mathrm{A}(\mathrm{t}), \mathrm{B}(\mathrm{t})$ and $\mathrm{C}(\mathrm{t})$. The cross-correlation coefficients between $\mathrm{A}, \mathrm{B}, \mathrm{C}$ and $\mathrm{E}$ have been verified to be $0 \pm 0.2$, and therefore the regression is well-posed.

Clear stimulus-related responses on the respiratory-asynchronous component $A(t)$ and on $\mathrm{B}(\mathrm{t})$ can clearly be observed. The responses obtained are compatible with optical observations, averaged over many stimuli, reported in the literature [19], superimposed to fluctuations that, in having verified on a scattering diffusing phantom (silicone matrix with aluminium oxide scattering particles, described elsewhere [25]) as being well above the instrumental noise, are compatible with spontaneous neural activity [11].

In order to rule out the possibility that the stimulus-synchronous responses may be due to artefacts, such as animal movements, we have analysed the signal obtained by displacing the source probe from the activated area, and by repositioning it in a surrounding region. We verify that a displacement of the probe a millimetre in any direction on the cortical surface from the expected activated cortical region is enough for any stimulus-synchronous response to disappear. We note that this is expected, and that the test was performed as a mere consistency check only as, under the deep myorelaxation 
associated with the anaesthesia protocol, any muscular contraction, including contractions synchronous with the stimulus, was de facto blocked.

In Figure 3 we report a typical response to a long stimulus pulse train. Again, clearly identifiable individual responses to the single stimuli can be observed, with consistent shape and timing, in zones $\mathrm{A}$ and $\mathrm{C}$ of the figure.

In such stimulus trains there have been repetitive fading episodes of the haemodynamic response, yet in the presence of stimuli (zone B of Figure 3), with a NIRS signal receding and then resurging with strength comparable to responsive epochs. The shift among waxing and waning epochs was often abrupt, and only occasionally gradual.

This alternate haemodynamic locking and unlocking to the stimulus may relate to the recently discovered family of complex discrepancies between the haemodynamic and the neuroelectric domains $[26,27]$ given that, in complementary experimental series with multiple single neuron recordings (data not shown here) neurons from the same cortical area responded faithfully, with intensity variations, to repetitive stimuli, without fading episodes.

As, in the current literature, individual optical responses to individual stimuli have never been isolated, this is the first experimental evidence of such patterns, and no further interpretation can be given at this time.

Because of the highly complex and finely distributed information decoding tasks, the brain shows high oxygen consumption rates and metabolic levels. The majority of cortical energy production is due to functional (synaptic) glutamatergic neuronal activity [28, 29]. In addition, it has been recently shown that metabolic rates in the central nervous system are the result of composite dynamic elements. Namely, a number of studies highlighted the crucial role of glia in the overall brain energetic balance [30]. It appears that glial cells act as interfaces between neurons and capillaries, activating complex intracellular 
metabolisms, e.g. through cyclooxygenase and prostaglandin cycles, regulating the microvessel diametre $[31,32]$.

Therefore, as it proceeds from the above issues, the cortical blood flow relies on a complex thread of metabolic and neurochemical pathways, conceptually remote from the mere expression of a haemodynamic survey. In addition, anaesthesia can influence the neurovascular coupling [33]. But, whereas studies on cognitive processes can be executed on awake animals, thus allowing for well-defined tasks and minimal perturbative factors, thus leading to a well-defined interpretation of results, e.g. subtle neurovascular processing discrepancies during task execution [34], in somatosensory cortex studies under general anaesthesia, the lack of awareness of the subject under examination constitutes a major obstacle.

Though Isoflurane anaesthesia influences the somatosensory primary responses [35], with tuned stimulation patterns [35] we can expect that the influence of anaesthesia on our recordings, if limited to the (mainly instrumental) scope of this paper, to be minor.

One of the most intricate points is due to the synthetic role of blood flow regulation on the many parameters that cooperate with the delicate timing synchronization to the oxygen, glucose and metabolite exchange in local microcircuits of the "tripartite synapses" [36]. Current imaging techniques on blood flow or on metabolic consumption privilege a single "argument" (e.g. oxygen consumption vs other parameters like glucose storing and metabolism).

One of the first goals to disentangle this complex picture implies the recording of the vascular response with critical adherence to fine temporal cues. Our method and our technical improvements allow indeed stringent time coherence to the trajectory of the stimulus delivery, gone unnoticed with previous techniques. This could truly represent a first step to achieve detailed understanding of brain "neuro-haemodynamics". 
Comparative work is being done in the literature on the matching between haemodynamic and neuroelectrical profiles. Significant results on whole systems have come from the comparative analyses of blood-oxygen-level-dependent (BOLD) signals and specific neuroelectrical profiles [37]. As, in time, BOLD-specific linear predictive modelling [38] has been superseded, nonlinear modelling has shown increasing complexity, first reporting vascular responses outlasting the saturation of electrical activities [20] and then, a dependence between neuronal activity and haemodynamic response exhibiting a saturating behaviour, and global linearity with embedded short-range nonlinearities [39]. Comparatively, an integration of fast neural dynamics into slow haemodynamics [40] has been observed and described, giving yet another variant on the interpretation of nonlinear neurovascular behaviours.

Even more recently, some groups have shown apparent discrepancies between haemodynamics and neural activity, demonstrating enhanced neural activity in the presence of active vasoconstriction [26] and a hitherto unknown anticipatory vascular response to tentative onset of neural signalling [27].

This scenario on BOLD responses, though still deserving strong cautions, may be potentially transferred to NIRS. Recent literature supports the homogeneity of NIRS and BOLD readings [41] and of their vascular sensitivity [42]. In offering an access to the cortical vascular dynamics to single and individual sensory stimuli with stringent temporal adherence, our system can be expected to yield an unprecedented fidelity to the nuances of the neural responses.

Further information can be derived from the observation that the respiratorysynchronous amplitude $\mathrm{C}(\mathrm{t})$ does not appear to exhibit clear stimulus-related responses such as $\mathrm{A}(\mathrm{t})$ ad $\mathrm{B}(\mathrm{t})$. Indeed, the technological accessibility of activity-related breath-tobreath and heartbeat-to-heartbeat differential oxygenation has been questioned as far back 
as 1998 [43]. The underlying picture [44], where analysis of the transcranial NIRS signal had shown components at respiratory frequency, left open the question whether such components were to be considered locally or systemically event-related. Indeed, our current measurements and analysis are suggestive of the latter case.

Seen from the side of the potential development of NIRS active implants, this indicates that while indeed signalling-related variations of tissue haemodynamics can be detected and timed, determination of the local oxygen consumption (and thus of the metabolic rate) from differential breath-to breath NIRS only, still proves to be challenging at the very least.

\section{CONCLUSIONS}

A system for the detection of the cortical NIRS recording of blood flow fluctuations, due to spontaneous or stimulus-induced conditions, optimized for simplicity and scalability to small size has been described. The system shows a response adequate both for reference data production and as a size- and complexity-compatible demonstrator for microengineered probes.

In providing a coherent and precisely timed single-stimulus response of local cortical haemodynamics, the system constitutes a novel pathway to the understanding of brain cortex physiology. The approach outlined has also given clear indications on the information that can, and cannot, be extracted from the breath-to-breath cerebral NIRS signal, thus providing substantial progress on resolving the issue whether the NIRS signal alone can be employed in a direct determination of cerebral oxygen consumption. 


\section{ACKNOWLEDGMENTS}

We wish to acknowledge support from Paolo Pedroli and Matteo Albertini in the fabrication of the probes and in the coding of the data analysis software, and from Dr. Maurizio Valente in the surgical preparation of the animals (anaesthesia and cannulations). 


\section{REFERENCES}

[1] V. Tuchin, Tissue Optics, SPIE, (Bellingham, 2000)

[2] F.F. Jöbsis, Science 198, 1264 (1977)

[3] H. Owen-Reece, M. Smith, C.E.Elwell and J.C. Goldstone, Br. J. Anaesth. 82, 418 (1999)

[4] S. Wray, M. Cope, D.T. Delpy, J.S. Wyatt and E.O.R. Reynolds, Biochim. Biophys. Acta 933, 184 (1988)

[5] M.E. Raichle and M.A.Mintun, Annu. Rev. Neurosci. 29, 449 (2006)

[6] S.B. Laughlin and T.J. Sejnowski, Science 301, 1870 (2003)

[7] D. Attwell and C. Iadecola, Trends Neurosci. 25, 621 (2002)

[8] S.B. Tekkök, A.M. Brown and B.R Ransom, J. Cereb. Blood Flow \& Metab. 23, $1340(2003)$

[9] H. Obrig, M. Neufang, R. Wenzel, M. Kohl, J. Steinbrink, K. Einhaupl and A. Villringer, NeuroImage 12, 623 (2000) 
[10] M.M. Plichta, M.J. Herrmann, A.C. Ehlis, C.G. Baehne, M.M. Richter and A.J. Fallgatter, Neuropsychobiology 53, 77 (2006)

[11] A. Aldo Faisal, L.P.J. Selen and D.M. Wolpert, Nature Rev. Neurosci. 9, 292 (2008)

[12] E.M.C. Hillman, J. Biomed. Opt. 12, 051402-1 (2007)

[13] J.N.D. Kerr and W. Denk, Nature Rev. Neurosci. 9, 195 (2008)

[14] ETG-4000, Hitachi Medical Systems, Japan

[15] Imagent, ISS Inc., USA

[16] A. Grinvald, D. Shoham, A. Shumel, D. Glaser, I. Vanzetta, E. Shtoyermann, H. Slovin, A. Sterkin, C. Wijnbergen, R. Hildesheim and A. Arieli, in: U. Windhorst and H. Johnasson (eds.), Modern Techniques in Neuroscience Research, chapter 34 (Springer Verlag, 1999)

[17] C.H. Petersen, T. Hahn, M. Mehta, A. Grinvald and B. Sakmann, Proc. Natl. Acad. Sci. 100, 1368 (2003)

[18] M. Nemoto, S. Sheth, M. Guiou, N. Pouratian, J.W.Y. Chen and A.W. Toga, J. Neurosci. 24, 3850 (2004) 
[19] J.J. Sable, D.M. Rector and G. Gratton, Eng. Med. Biol. Mag. 26 (iss. 4), 17 (JulAug 2007)

[20] A. Devor, A. K. Dunn, M. L. Andermann, I. Ulbert, D. A. Boas and A. M. Dale, Neuron 39, 353 (2003)

[21] W.J. Witterman, Detection and Signal Processing, Chapters 7-8 (Springer, 2006)

[22] M. Zimmermann. Pain 16, 109 (1983)

[23] G. Paxinos and C. Watson, The Rat Brain in Stereotaxic Coordinates (Academic Press, 2007)

[24] W.H. Press, S.A. Tekulosky, W.T. Vetterling and B.P. Flannery, Numerical Recipes in C (Second Edition), chapter 15 (Cambridge Universty Press, 1992)

[25] M.E. Giardini, L. loconte, G. Guizzetti. A. Gelmetti and P. Lago, SPIE Proc. 3568, $59(1998)$

[26] A. Devor, E.M.C. Hillman, P. Tian, C. Waeber, I.C. Teng, L. Ruvinskaya, M.H. Shalinsky, H. Zhu, R.H. Haslinger, S.N. Narayanan, I. Ulbert, A.K. Dunn, E.H. Lo, B.R. Rosen, A.M. Dale, D. Kleinfeld and D.A. Boas, J. Neurosci. 28, 14347 (2008)

[27] Y.B. Sirotin and A. Das, Nature 457, 475, 2009 
[28] A. Jeromin, L.L. Yuan, A. Frick, P. Pfaffinger, and D. Johnston, J. Neurophysiol. 90, $2741(2003)$

[29] N. R. Sibson and A. Dhankhar, Proc. Natl. Acad. Sci. USA 95, 316 (1998)

[30] P.J. Magistretti, J. Exp. Biol. 209, 2304 (2003)

[31] M. Zonta, A. Sebelin, S. Gobbo, T. Fellin, T. Pozzan and G. Carmignoto, J. Physiol. 553, 407 (2004)

[32] S.J. Mulligan and B.A. MacVicar, Nature 431, 195 (2004)

[33] K. Sicard, Q. Shen, M.E. Brevard, R.Sullivan, C.F. Ferris, J.A. King and T.Q. Duong, J. Cereb. Blood Flow \& Metabol. 23, 472 (2003)

[34] J. Fuster, T.M. Guiou, A. Ardestani, A. Cannestra, S. Sheth, Y.D. Zhou, A. Toga and M. Bodner, NeuroImage 26, 215 (2005)

[35] K. Masamoto, T. Kim, M. Fukuda, P. Wang and S.G. Kim, Cerebral Cortex 17, $942(2007)$

[36] M.M. Halassa, T. Fellin and P.G. Haydon, Trends Mol. Med. 13, 54 (2007) 
[37] N.K. Logothetis and B.A.Wandell, Ann. Rev. Physiol. 66, 735 (2004)

[38] M.D. Robson, J.L. Dorosz and J.C.Gore, Neuroimage 7, 85, (1998) (Erratum: Neuroimage 8, 228 (1998))

[39] S.A. Sheth, M. Nemoto, M. Guiou, M. Walker, N. Pouratian and A.W. Toga, Neuron 42, 347 (2004)

[40] X. Wan, J. Riera, K. Iwata, M. Takahashi, T. Wakabayashi and R. Kawashima, NeuroImage 32, 616 (2006)

[41] A. Sassaroli, B. de B Frederick, Y. Tong, P.F. Renshaw and S.Fantini, Neuroimage 33, 505 (2006)

[42] T.J. Huppert, R.D. Hoge, S.G. Diamond, M.A. Franceschini and D.A. Boas, Neuroimage 29, 368 (2006).

[43] U.J. Scholz, M. Kohl, C. Nolte, C. Hening and A. Villringer, SPIE Proc. 3566, 106 (1998)

[44] E. Chihara, T. Shiga, K. Tanabe and Y. Tanaka, SPIE Proc. 3194, 83 (1997) 


\section{SHORT CVs}

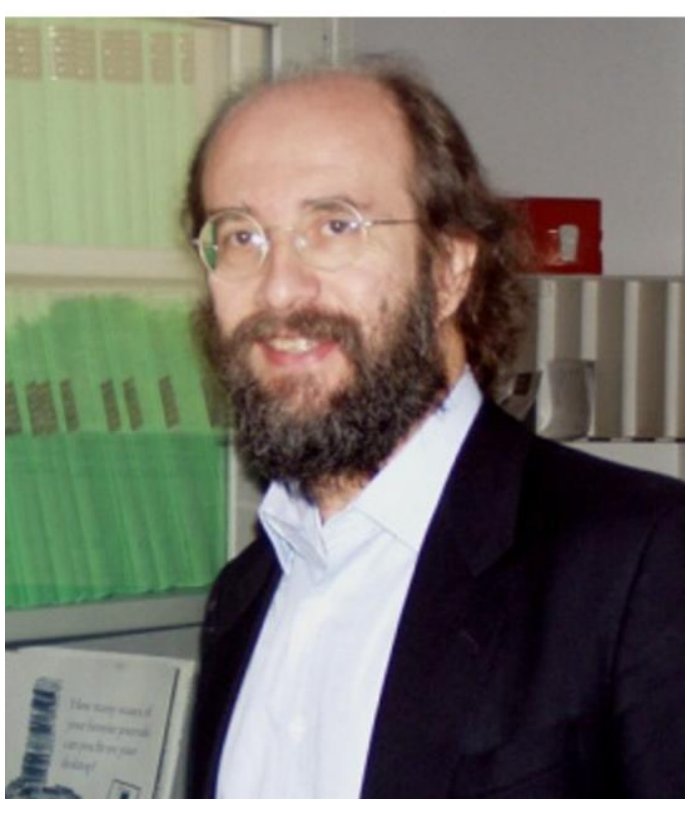

Gabriele Bella obtained the Degree in Medicine and Surgery in 1979 at the Faculty of Medicine at the University of Milan, and specialized in Neurological Sciences in 1984 at the same Institution. In 1984 became assistant professor at the Chair of Physiopathology of Pain at the University of Milan. In 2001 he was appointed Head of Research at the Institute of Molecular Bioimaging and Physiology of the National Research Council. He is an experimental neurophysiologist and electrophysiologist, interested in the processing of sensory signals in the spinal cord, the thalamus and the somatosensory cortex. He specifically addresses his studies to the neurophysiological and electrophysiological evaluation of chronic pain, using different animal experimental models, with neuronal single unit and multitrace multiple electrode electrophysiology, and with EEG techniques. His interest extends to electrooptical techniques for the study of thalamo-cortical processing of sensory signals. 


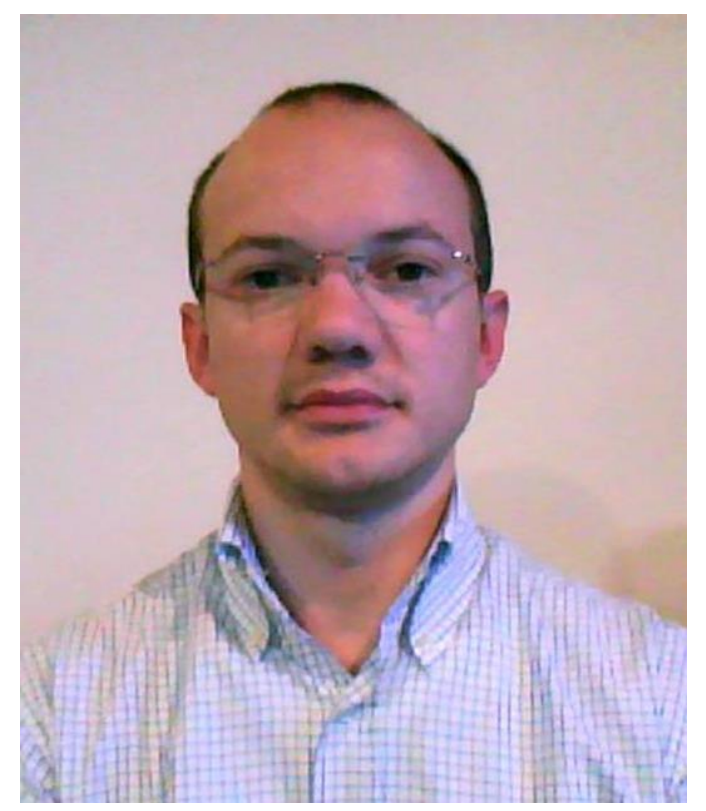

Stefano Trevisan studied Electronic Engineering at the University of Pavia, Italy, graduating in 2003. He completed his studies in 2007 with a Doctorate in Electronic Engineerig and Computer Science, within the same University and collaborating with Mario Giardini in the National Institute for the Physics of Matter (INFM), working, amongst other topics, on infrared spectroscopy techniques for the monitoring of cerebral activity. His research activity has been focussed on the study of brain haemodynamics both in basal conditions and in response to nociceptive stimuli. He currently holds an industrial position as Project Manager in a laser source manufacturing company. 


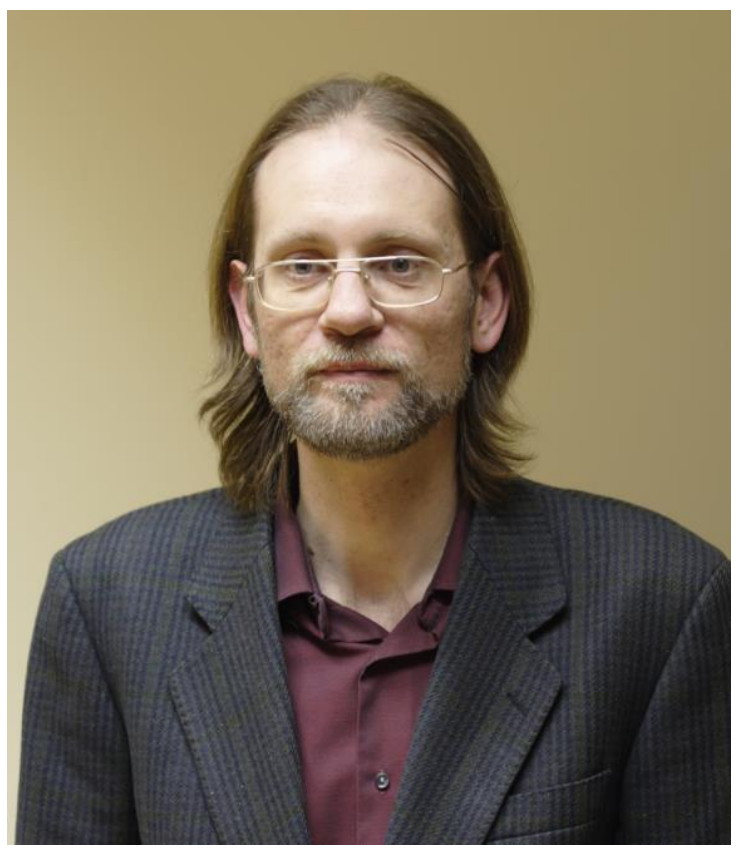

Mario Giardini graduated in 1991 and received his $\mathrm{PhD}$ in Electronic Engineering and Computer Science in 1994 at the University of Pavia, Italy. In 1997 he became Research Scientist in the National Institute for Physics of Matter (INFM, Italy) where, from 2003, he has been in charge of the Technology Transfer division of the INFM Laboratory of Physics for Medicine. He has developed instruments, devices and technologies for the most diverse applications, mainly dedicated to the biomedical and biotechnological world, and he has authored academic papers and patents relating to in-vivo and in-vitro optical analysis and detection, to fabricative processes and to medical devices. His academic research has implied a strong contact with Industry, where from 2005 to 2008 he has headed the R\&D activities of a leading European microscope manufacturer. Since January 2008, he has joined as Research Fellow the University of St Andrews, United Kingdom. 


\section{FIGURE CAPTIONS}

Figure 1 - The detector (a) and source (b) probes, showing the optical fibre pigtailed to a photodiode and a LED respectively, interposing an optical matching gel/adhesive. The thermorestringent tubing encapsulating the photodiode/LED-to-fibre joint is not shown for clarity. In (c) a simplified diagram of the photodiode preamplifier.

Figure 2 - The raw signal, as detected (a), and the traces $\mathrm{A}(\mathrm{t}), \mathrm{B}(\mathrm{t})$ and $\mathrm{C}(\mathrm{t})$ extracted from regression. (b) and (c) show 5 spikes synchronous with 5 stimulation pulses, whose delivery timing is indicated with dotted lines. The expanded section of trace (a) visually highlights the respiratory-synchronous component that dominates the raw signal.

Figure 3 - A typical A(t) response to a long sequence of stimuli (vertical dotted and full lines). In regions (a) and (c) we observe clear response peaks, while in (b), though the presence of the stimuli, such peaks are absent. 
Figure 1

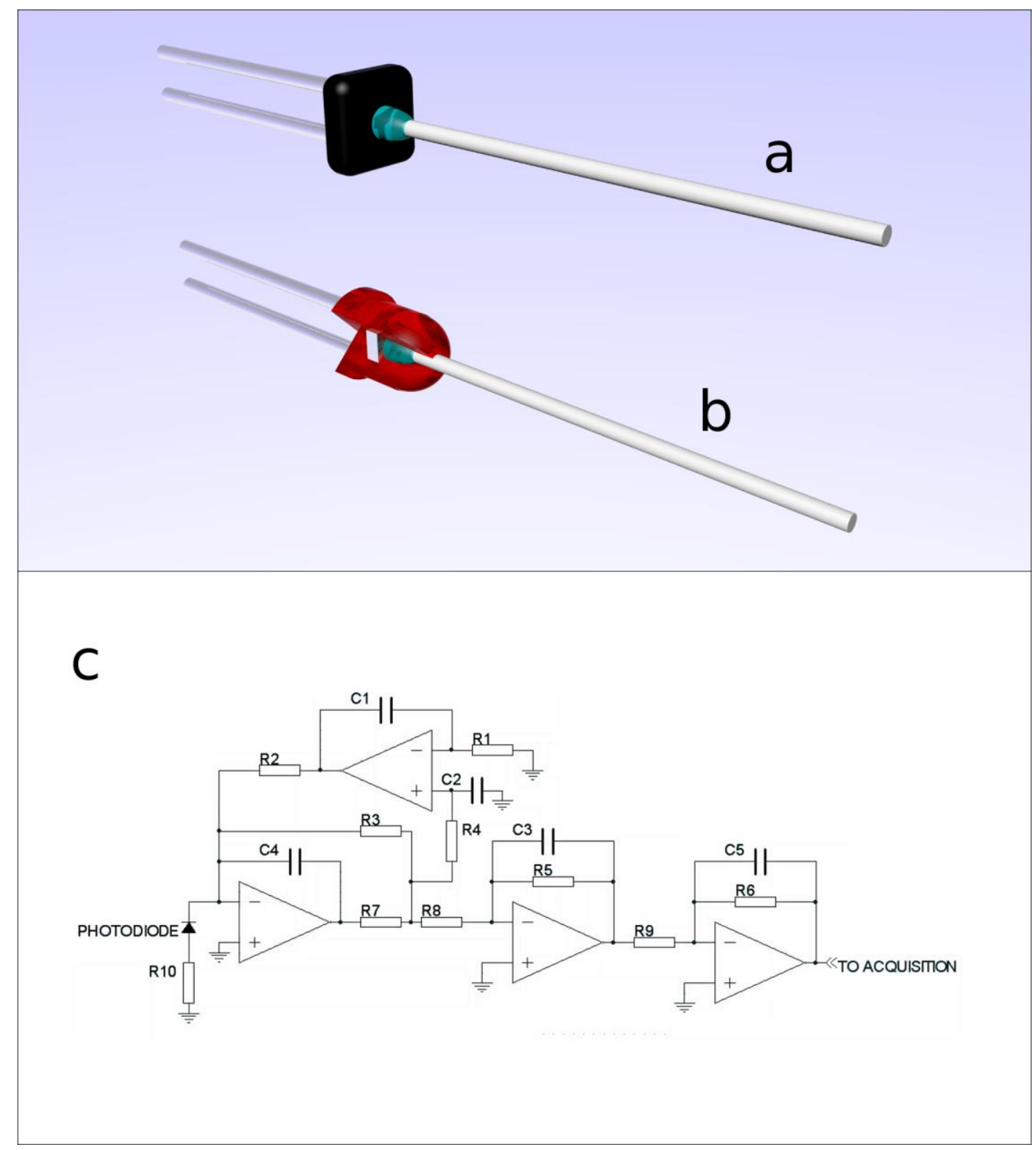

Page 29 of 31 
Figure 2

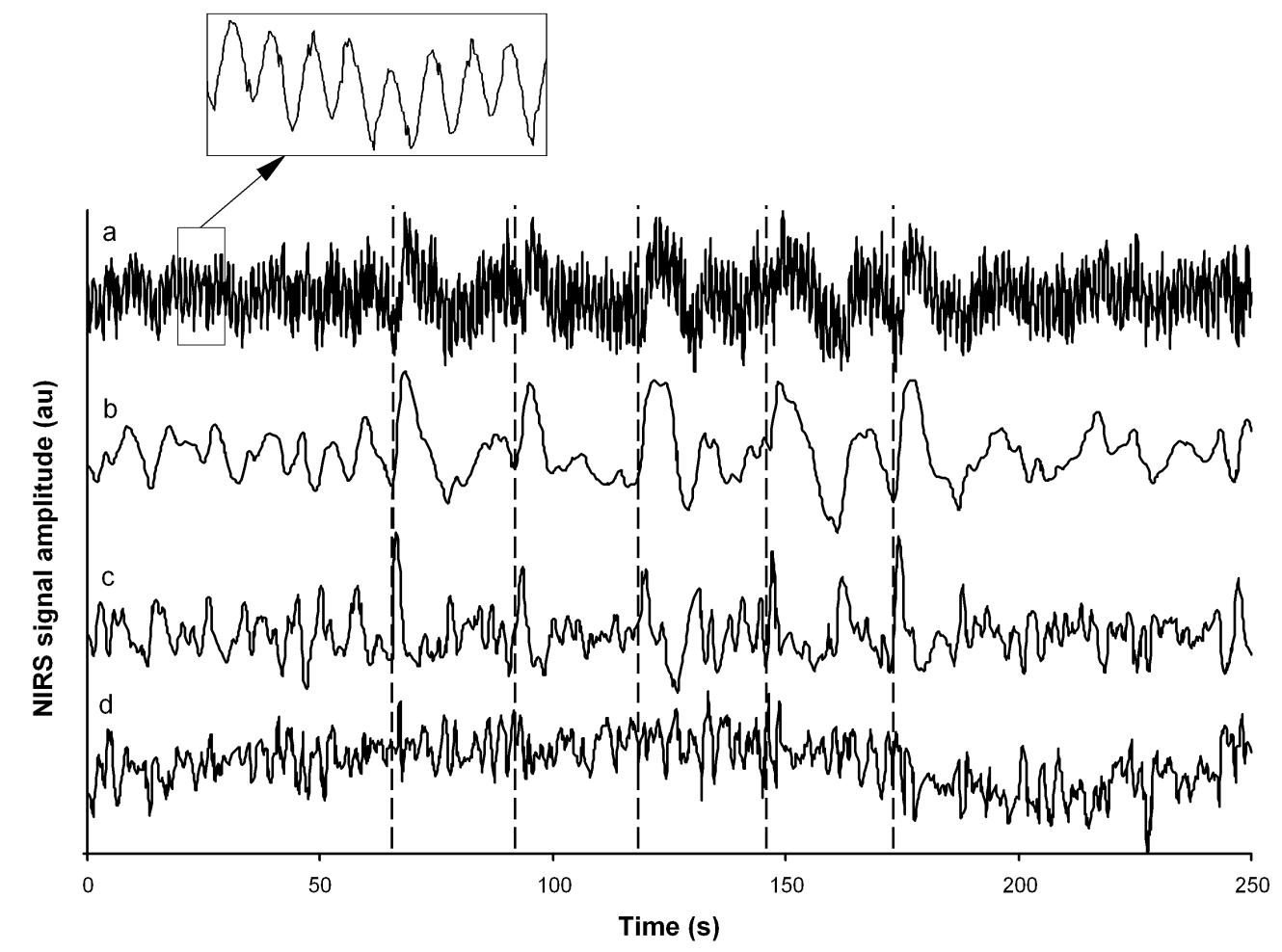


Figure 3

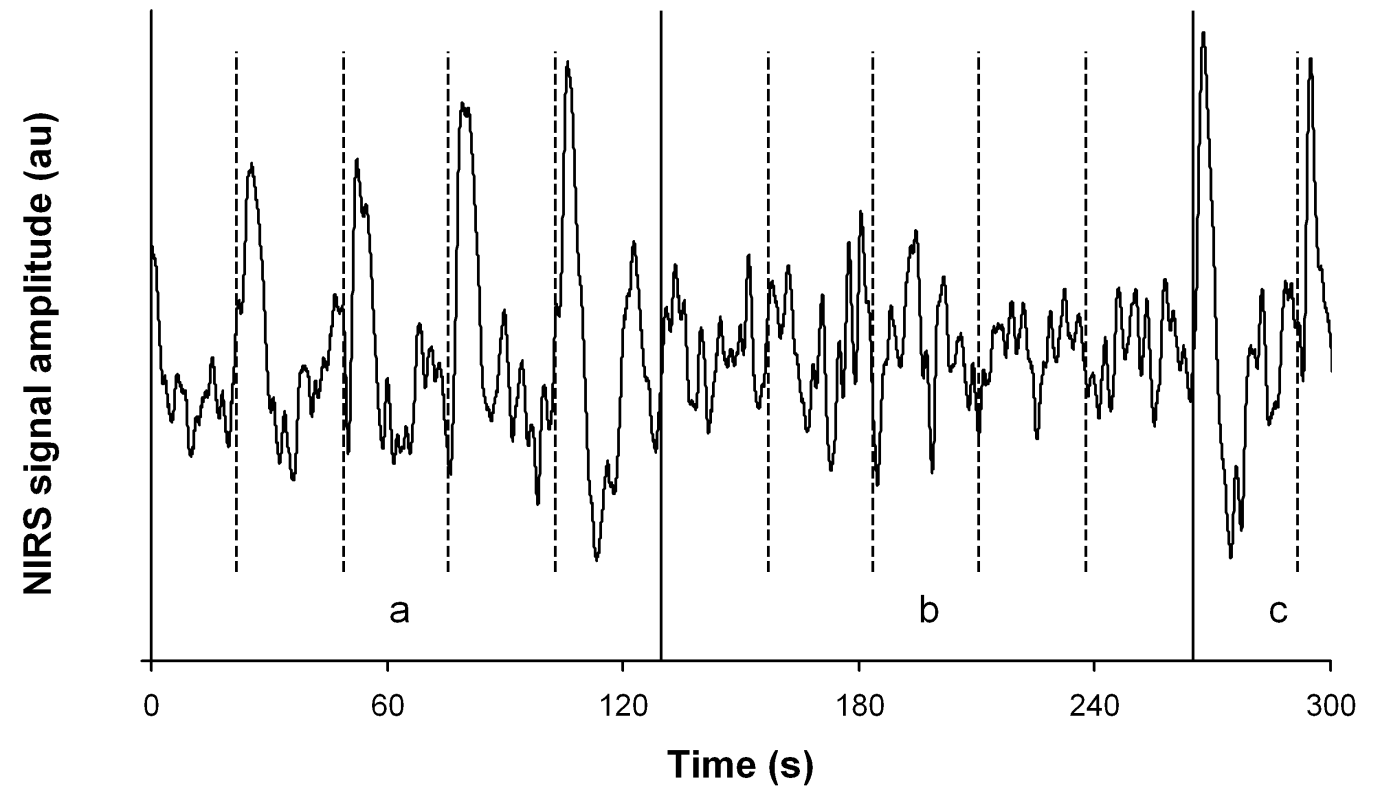

Page 31 of 31 